\title{
Safety and Efficacy of Intravenous Tirofiban as Antiplatelet Premedication for Stent-Assisted Coiling in Acutely Ruptured Intracranial Aneurysms
}

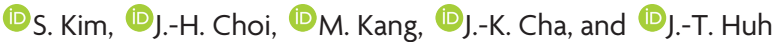

\begin{abstract}
BACKGROUND AND PURPOSE: Stent-assisted coiling of intracranial aneurysms requires antiplatelet therapy, typically aspirin and clopidogrel to prevent thromboembolic complications. There is a substantial concern that tirofiban may increase the risk of hemorrhage when used as an antiplatelet premedication in ruptured intracranial aneurysms. Our aim was to evaluate the safety and efficacy of intravenous tirofiban administration, instead of oral dual antiplatelet agents, as an antiplatelet premedication for stent-assisted coiling in patients with acutely ruptured intracranial aneurysms.
\end{abstract}

MATERIALS AND METHODS: We conducted a retrospective review of a data base containing a consecutive series of patients who underwent stent-assisted coiling for acutely ruptured intracranial aneurysms between March 2010 and January 2015. Intravenous tirofiban was administered to all patients before stent-assisted coiling, instead of premedication with loading doses of aspirin or clopidogrel.

RESULTS: Forty patients with 41 aneurysms received intravenous tirofiban and underwent stent-assisted coiling. None of the patients had a newly developed intracerebral hemorrhage, subarachnoid hemorrhage, or intraventricular hemorrhage. Intraprocedural aneurysmal rupture occurred in 2 patients (5\%). Cerebral infarction developed in 2 patients (5\%). Ventriculostomy-related hemorrhage was seen in 2 of 10 patients in whom ventriculostomy was performed before or after coiling. Thirty-four (85\%) patients had a good outcome (Glasgow Outcome Score of 4 or 5) at the time of discharge, but 1 patient died of cardiac arrest. None of the patients developed thrombocytopenia, retroperitoneal, gastrointestinal, or genitourinary bleeding related to tirofiban administration.

CONCLUSIONS: In our study, tirofiban showed a low risk of symptomatic hemorrhagic or thromboembolic complications. Tirofiban may offer a safe and effective alternative as an antiplatelet premedication during stent-assisted coiling of acutely ruptured intracranial aneurysms.

ABBREVIATIONS: $E V D=$ external ventricular drain; GOS = Glasgow Outcome Score; $\mathrm{HH}=$ Hunt and Hess

$\mathbf{R}$ esults from the International Subarachnoid Aneurysm Trial showed that the endovascular management of intracranial aneurysm is a safe, effective, and sometimes preferable treatment option. ${ }^{1}$ However, endovascular treatment of ruptured wide-neck aneurysms is still a challenge to neurointerventionalists because of the controversy surrounding the use of stent placement as an adjuvant therapy for the coiling of acutely ruptured aneurysms, due to the need for antiplatelet

Received June 17, 2015; accepted after revision August 13.

From the Departments of Radiology (S.K., M.K.), Neurosurgery (J.-H.C., J.-T.H.), and Neurology (J.-K.C.), Busan-Ulsan Regional Cardio-Cerebrovascular Disease Center, Dong-A University Hospital, Busan, Republic of Korea.

Sanghyeon Kim and Jae-Hyung Choi are co-first authors.

This work was supported by the Dong-A University Research Fund.

Please address correspondence to Myongjin Kang, MD, Department of Radiology, Busan-Ulsan Regional Cardio-Cerebrovascular Disease Center, Dong-A University Hospital, 26 Daesingongwon-ro, Seo-gu, Busan 602-715, Republic of Korea; e-mail: myongjin.kang@gmail.com

http://dx.doi.org/10.3174/ajnr.A4551 medications. Stent-assisted procedures are particularly prone to thromboembolic complications, with a reported rate of thromboembolic events of $7 \%-15 \%$ during stent-assisted coiling. ${ }^{2-4}$ Therefore, there is a need for preoperative antiplatelet therapy with optimal anticoagulation during the procedures, even with subarachnoid hemorrhage. However, there is no consensus about when and how patients should be loaded with antiplatelet medication before the procedure.

Glycoprotein IIb/IIIa antagonists have attracted attention for the prevention or treatment of thromboembolism during coiling of intracranial aneurysms, ${ }^{5-7}$ but the increased risk of intracranial hemorrhage following glycoprotein IIb/IIIa inhibition remains a substantial concern. Moreover, the safety and efficacy data regarding the use of tirofiban in endovascular aneurysm treatment are lacking.

The objective of our study was to evaluate the safety and efficacy of intravenous tirofiban, instead of clopidogrel and aspirin, as an antiplatelet premedication for stent-assisted coiling in patients with acutely ruptured intracranial aneurysms. 


\section{MATERIALS AND METHODS}

\section{Patients}

We conducted a retrospective review of a neurointerventional data base containing data collected from a consecutive series of patients who underwent stent-assisted coiling of acutely ruptured intracranial aneurysms between March 2010 and January 2015. SAH was diagnosed by using noncontrast brain CT.

The clinical severity of SAH was assessed by using the Hunt and Hess $(\mathrm{HH})$ grading system, and the radiographic grade was classified by using the Fisher grade. Clinical outcomes were assessed at discharge by using the Glasgow Outcome Score (GOS) and classified as follows: 1) dead; 2) vegetative state; 3 ) severely disabled; 4) moderately disabled; and 5) mildly or not disabled. A GOS of $\geq 4$ was considered a good clinical outcome. Medical charts were also reviewed to determine clinical or subclinical worsening of radiologic findings after the procedures and to evaluate any possible systemic complications related to tirofiban therapy such as retroperitoneal, gastrointestinal, or genitourinary bleeding and thrombocytopenia.

\section{Procedures}

The decision to use stent-assisted coiling was based on aneurysm morphology, such as a wide-neck aneurysm, defined by a fundus-toneck ratio of less $<2$, a neck diameter of $\geq 4 \mathrm{~mm}$, or the need for stent placement after intraprocedural coil prolapse. All procedures were performed with written informed consent. In patients with SAH, the diagnostic angiogram and the coiling procedure were performed on the day of admission. All patients underwent endovascular treatment during a single session under general anesthesia.

Despite the use of aggressive intraoperative antiplatelet therapy, angiographic catheters are contact activators of the coagulation cascade and are significant sources of thromboembolic complications. ${ }^{8}$ Thus, to prevent this complication, a solution of 5000 IU of heparin in $1000 \mathrm{~mL}$ of $0.9 \%$ normal saline was administered continuously through the guiding catheter.

Intravenous tirofiban was administered to all patients, instead of premedication with loading doses of aspirin or clopidogrel, before stent-assisted coiling. The intravenous tirofiban infusion was initiated only after confirming that contrast was not filling the dome (presumed rupture site) of the aneurysm. Patients received a $0.4-\mu \mathrm{g} /$ $\mathrm{kg} / \mathrm{min}$ loading dose of tirofiban for 30 minutes followed by a 0.10 $\mu \mathrm{g} / \mathrm{kg} / \mathrm{min}$ maintenance infusion. A loading dose of clopidogrel and aspirin (300 mg each) was administered after the tirofiban maintenance infusion, followed by daily doses of $75 \mathrm{mg} /$ day of clopidogrel for 6 months and $100 \mathrm{mg} /$ day of aspirin indefinitely.

We used the "jailing" technique in all patients. The microcatheter was initially positioned within the aneurysm and then coiled within the aneurysm until contrast was no longer filling the dome of the aneurysm; then tirofiban infusion was initiated. The stent was deployed 15 minutes after the initiation of tirofiban administration, and the aneurysm was packed with more coils after stent deployment. Aneurysms were embolized with a variety of bare platinum coils at the operator's discretion.

Stent-assisted coiling was performed by using a Neuroform stent (Stryker Neurovascular, Kalamazoo, Michigan), Enterprise stent (Codman \& Shurtleff, Raynham, Massachusetts), or
Table 1: Clinical and radiologic characteristics of patients

\begin{tabular}{lc}
\multicolumn{1}{c}{ Characteristics } & No. of Cases (\%) \\
\hline Mean age (yr) (range) & $57.3 \pm 10.5(41-84)$ \\
Sex (M/F) & $12: 28$ \\
Hunt and Hess grade & \\
1 & $5(12.5)$ \\
2 & $22(55)$ \\
3 & $8(20)$ \\
4 & $5(12.5)$ \\
5 & 0 \\
Fisher grade & \\
1 & $2(5)$ \\
2 & $10(25)$ \\
3 & $15(37.5)$ \\
4 & $13(32.5)$ \\
Clinical outcome (GOS) & \\
1 & $1(2.5)$ \\
2 & $1(2.5)$ \\
3 & $4(10)$ \\
4 & $4(10)$ \\
5 & $30(75)$ \\
\hline
\end{tabular}

Solitaire stent (Covidien, Irvine, California). The choice of stent was made on a case-by-case basis according to the operator's preference, anatomic considerations, and specific clinical situations.

All patients underwent brain CT immediately after the procedure to ascertain any new or worsening SAH or intraparenchymal hemorrhage during the acute phase of tirofiban infusion. An external ventricular drain (EVD) was placed in patients experiencing clinical deterioration, in whom developing hydrocephalus was confirmed by follow-up brain CT. Tirofiban was not stopped during the EVD operation.

Postprocedural hemorrhage and infarction were evaluated with brain CT and/or MR imaging. All patients underwent brain CT immediately after the procedure and were followed up for 7 days. Patients were re-evaluated if their clinical condition worsened during hospitalization, and they underwent additional brain $\mathrm{CT}$ and/or MR imaging to determine the cause of their deterioration. Cerebral infarction was evaluated depending on the vascular territory involved and the timing of the appearance of neurologic symptoms. The effect of vasospasm due to SAH was also considered.

\section{RESULTS}

Forty patients with 41 aneurysms underwent stent-assisted coiling and received intravenous tirofiban. Of those, 28 (70\%) were women and $12(30 \%)$ were men, with a mean age of $57.3 \pm 10.5$ years (range, $41-84$ years). In terms of clinical severity, 5 (12.5\%), $22(55 \%), 8(20 \%)$, and $5(12.5 \%)$ patients had HH grades of 1,2, 3 , and 4 , respectively. The clinical and radiologic characteristics of patients are summarized in Table 1.

The mean aneurysm size was $5.9 \pm 2.9 \mathrm{~mm}$ (range, $2-13 \mathrm{~mm}$ ). The location of the aneurysms was the anterior communicating artery in 17 aneurysms, posterior communicating artery in 11 aneurysms, internal carotid artery in 4 aneurysms, vertebrobasilar artery in 4 aneurysms, middle cerebral artery in 3 aneurysms, and anterior cerebral artery in 2 aneurysms. Solitaire, Neuroform, and Enterprise stents were used in 25 (62.5\%), 11, and 4 patients, respectively. 


\section{Complications}

None of the patients showed a newly developed intracerebral hemorrhage, $\mathrm{SAH}$, or intraventricular hemorrhage on immediate follow-up brain CT after coiling (Table 2).

Intraprocedural aneurysmal rupture occurred in 2 patients (Fig 1) as a result of coil extrusion during coil insertion, after tirofiban injection and stent deployment in 1 patient and by the high tension of the microcatheter before tirofiban injection in the other. In the former patient, we packed the aneurysm with more coils until the bleeding stopped while maintaining tirofiban administration. In the latter, the aneurysm was packed with more coils until the bleeding stopped, but the coils protruded into the parent artery. Thus, we deployed a stent after tirofiban infusion. These 2 cases showed slight worsening of SAH on a brain CT scan performed immediately after the procedure, but patients were discharged without neurologic deficits.

An EVD was placed in 10 patients. Of those, the EVD was placed before the stent-assisted coiling in 5 patients who showed hydrocephalus in the initial brain CT. Ventriculostomy-related hemorrhage was seen in 2 of 5 patients (Fig 2), but the hemorrhage was small and located along the catheter tract. Further follow-up brain CT demonstrated resolution of the hemorrhage.

EVDs were placed on the same day in the other 5 patients who did not show hydrocephalus on the initial brain CT but developed hydrocephalus after stent-assisted coiling. No surgical difficulties or ventriculostomy-related hemorrhages resulting from an increased bleeding tendency due to tirofiban therapy were observed. Of the 10 patients who had undergone EVD, 1 patient underwent EVD after stent-assisted coiling and required permanent CSF di-

\section{Table 2: Complications}

\begin{tabular}{ll}
\hline \multicolumn{1}{c}{ Complications } & No. of Cases (\%) \\
\hline Hemorrhagic complications & \\
Newly developed ICH, SAH, or IVH & $0(0)$ \\
Intraoperative rupture & $2(5)$ \\
Ventriculostomy-related hemorrhage & $2(20)^{\mathrm{a}}$ \\
Infarction & $2(5)$ \\
\hline
\end{tabular}

Note:- $\mathrm{ICH}$ indicates intracerebral hemorrhage; IVH, intraventricular hemorrhage. a Percentages were obtained in relation to the number of patients in whom ventriculostomy was placed before or after coiling $(n=10)$, whereas other complications are over the total number of patients $(n=40)$.
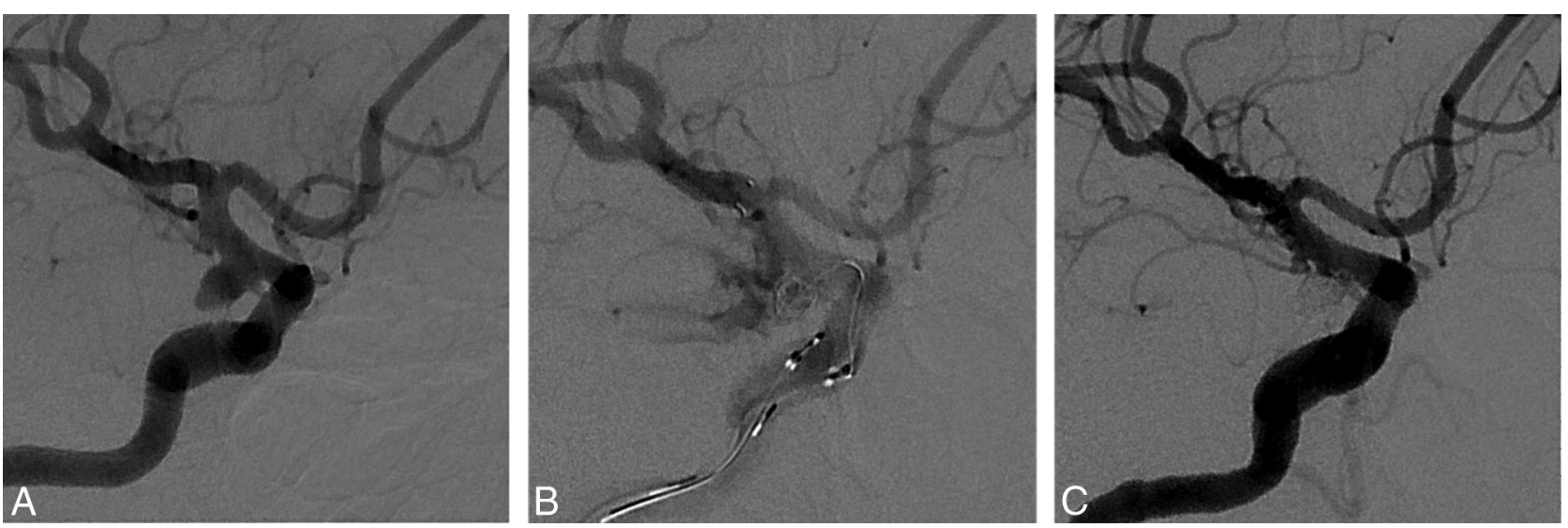

FIG 1. A 53-year-old woman presented with a Hunt and Hess grade 1 subarachnoid hemorrhage. $A$, Digital subtraction angiography shows a wide-neck right posterior communicating artery aneurysm of approximately $15.49 \times 3.72$ mm. $B$, Angiography shows contrast extravasations during coil insertion after tirofiban injection and stent deployment. We packed the aneurysm with more coils while maintaining tirofiban. $C$, Immediate posttreatment angiography shows cessation of contrast extravasations and complete occlusion of the aneurysm. 
stent placement. Therefore, given the thrombogenicity of endovascular stents, anticoagulation is recommended in patients undergoing stent-assisted procedures to prevent thromboembolic
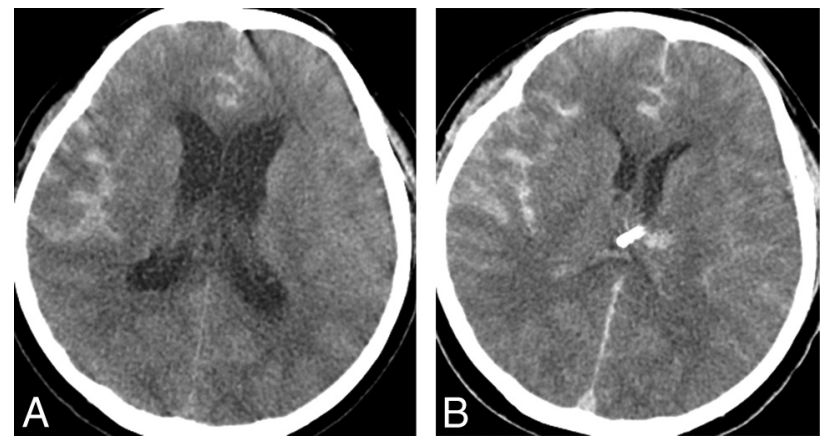

FIG 2. A 54-year-old woman presented with a Hunt and Hess grade 4 subarachnoid hemorrhage. $A$, Initial precontrast brain CT scan shows subarachnoid hemorrhage and hydrocephalus. An external ventricular drain was placed before stent-assisted coiling. $B$, Immediate posttreatment precontrast brain CT scan shows asymptomatic ventriculostomy-related hemorrhage. The hemorrhage was small and occurred along the catheter tract. A 7-day follow-up brain CT scan (not shown in Fig 2) shows disappearance of the hemorrhage.
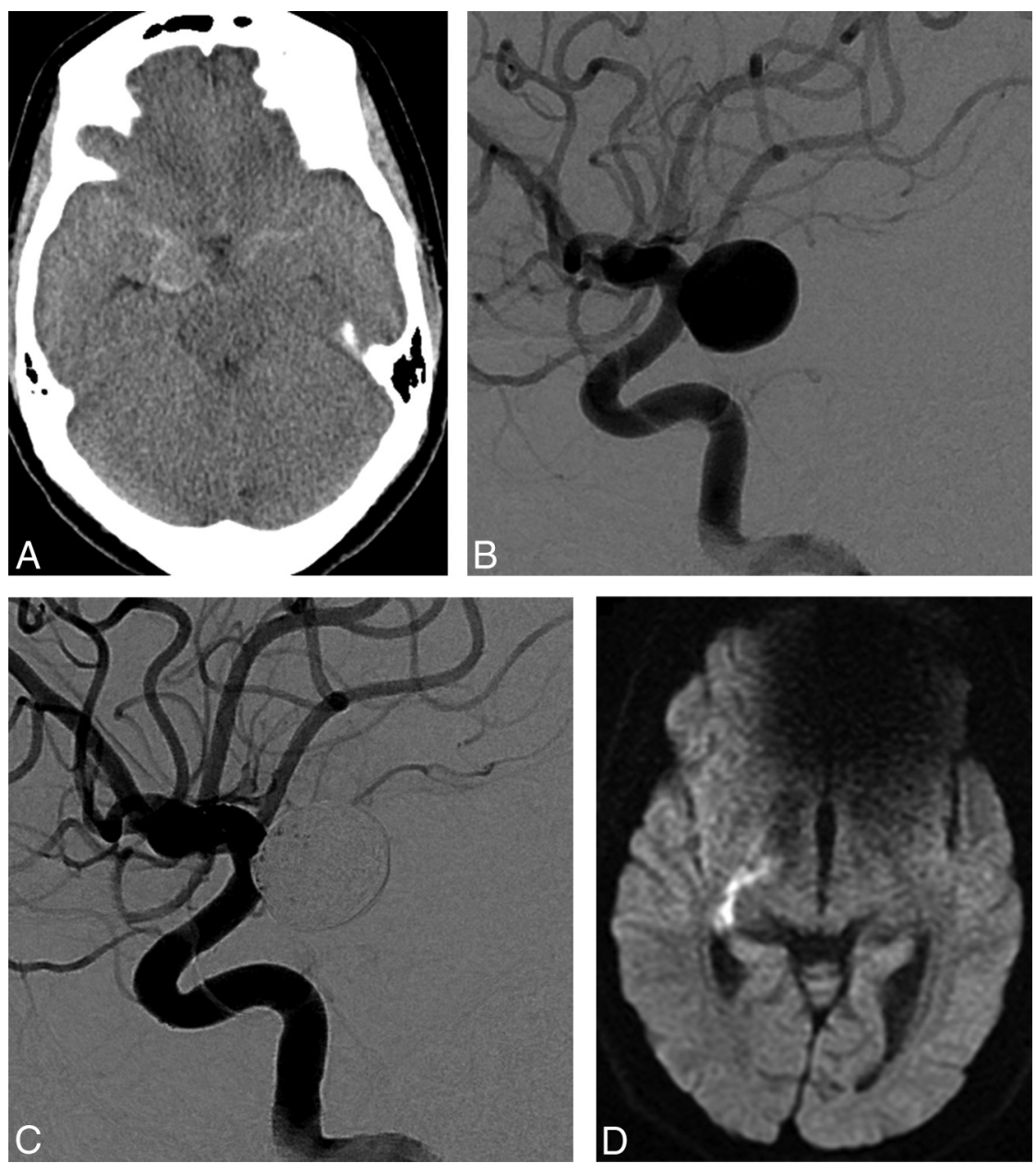

FIG 3. A 44-year-old woman presented with a Hunt and Hess grade 2 subarachnoid hemorrhage. $A$, Initial precontrast brain CT scan showed subarachnoid hemorrhage. B, Digital subtraction angiography shows a wide-neck right posterior communicating artery aneurysm of approximately $11.3 \times 12 \mathrm{~mm}$. C, Immediate posttreatment angiography shows complete occlusion of the aneurysm and normal patency of the anterior choroidal artery. D, Diffusion-weighted MR imaging shows infarction of the posterior limb of the right internal capsule on postoperative day 7 . events such as in-stent thrombosis. Intra- and postprocedural anticoagulation with heparin and antiplatelet therapy with aspirin or clopidogrel are widely used and reduce thromboembolism rates in patients undergoing coiling for cerebral aneurysms. ${ }^{10-12}$ However, antiplatelet drugs take time to reach therapeutic levels. For example, a 600-mg loading dose of clopidogrel provides platelet inhibition of $55 \%-59 \%$ within 4 hours after administration. ${ }^{13}$

In the setting of SAH, the use of a stent is not always anticipated because the decision to place a stent is sometimes made during the procedure and pretreatment with antiplatelet drugs is not always possible. In our center, the diagnostic angiogram and the coiling procedure are performed on the day of admission to minimize the risk of rebleeding in patients with SAH. Hence, the necessity of a stent during coiling cannot be anticipated in these patients; this scenario precludes premedication with aspirin or clopidogrel. Thus, intravenous tirofiban was injected intraprocedurely in all patients to prevent thromboembolic complications, instead of aspirin and clopidogrel, before stent-assisted coiling of acutely ruptured intracranial aneurysms.

Available glycoprotein IIb/IIIa inhibitors include abciximab, tirofiban, and eptifibatide. Abciximab is more commonly used than tirofiban to treat patients with acute coronary syndrome. However, several studies reported that abciximab and eptifibatide may induce fatal intracerebral hemorrhage. ${ }^{7,14-16}$ Moreover, abciximab binds irreversibly to the glycoprotein IIb/IIIa receptor causing platelet-function suppression for almost 48 hours after interruption of the infusion. ${ }^{17}$

Tirofiban acts as a reversible antagonist of fibrinogen by binding to the glycoprotein IIb/IIIa receptor on platelets. Tirofiban has a rapid onset of action (5 minutes). ${ }^{18}$ When administered according to the regimen of $0.4 \mu \mathrm{g} / \mathrm{kg} / \mathrm{min}$ for 30 minutes followed by a $0.1-\mu \mathrm{g} / \mathrm{kg} / \mathrm{min}$ maintenance infusion, $>90 \%$ inhibition of platelet aggregation is attained by the end of the initial 30-minute infusion. Its plasma half-life is 2 hours, and following discontinuation of an infusion of tirofiban, $0.10 \mu \mathrm{g} / \mathrm{kg} / \mathrm{min}$, ex vivo platelet aggregation returns to near baseline in 4-8 hours in approximately $90 \%$ of patients. ${ }^{14,19}$

Experience with tirofiban in the neuroendovascular setting has been limited. Chalouhi et $\mathrm{al}^{20}$ assessed the safety and efficacy of tirofiban in stent-assisted coiling as a prophylactic treatment and as a rescue therapy for thromboembolic events. They reported that the incidence of tirofiban-related hemorrhage and mortality in patients treated with a bolus followed by a maintenance protocol was as high as $18.8 \%$ and $12.5 \%$, respec- 
tively. However, the study enrolled patients who were presenting with not only ruptured aneurysms but also unruptured aneurysms. Furthermore, in contrast to our study, all patients with aneurysms treated in the setting of SAH were loaded with 600 $\mathrm{mg}$ of clopidogrel and $325 \mathrm{mg}$ of aspirin intraprocedurally and infused with tirofiban immediately after stent deployment or placement of the first coil. In this study, we assessed the safety and efficacy of intravenous tirofiban without aspirin and clopidogrel administration as a premedication in stent-assisted coiling of ruptured aneurysms.

In the immediate postrupture period, further bleeding from the aneurysm dome is prevented by a fibrin-platelet plug. On the basis of their pharmacologic properties, glycoprotein IIb/IIIa receptor antagonists may promote breakdown of hydrogen sulfate bonds between platelets, leading to lysis of the platelet-rich clot/ thrombus, which then increases the chance of intraprocedural rupture. ${ }^{21}$ Furthermore, in the event of iatrogenic aneurysm perforation, the likelihood of a devastating hemorrhage is also increased. In our study, intraprocedural aneurysmal rupture occurred in 2 cases before or after tirofiban infusion and stent deployment. Bleeding was stopped after packing the aneurysm with extra coils. Immediate postprocedural brain CT scans showed slight worsening of the subarachnoid hemorrhage, but patients were discharged without neurologic deficits.

The coexistence of acute hydrocephalus at the time of presentation is an additional complicating factor when considering stent-assisted coiling following $\mathrm{SAH}$. Hemorrhage rates after EVD placement varied widely from $1 \%$ to $33 \%{ }^{22-24}$ Scholz et $\mathrm{al}^{25}$ reported a hemorrhage rate of $14.8 \%$ in patients who underwent EVD placement after endovascular coil embolization of cerebral aneurysms under anticoagulation or antiplatelet therapy. The risk of ventriculostomy-related hemorrhage is higher in patients with SAH treated with stent-assisted coiling than in those with coiling without a stent. ${ }^{26}$

In this study, an EVD was placed in 10 of 40 patients, with ventriculostomy-related hemorrhage occurring in 2 of 5 patients whose EVD was placed before coiling. The hemorrhage was characterized by small petechial bleeding along the catheter tract, which was $<1 \mathrm{~cm}$ in diameter, asymptomatic, and temporary. Five patients underwent an additional EVD placement within 1 day without stopping tirofiban after stent-assisted coiling. There were no operational complications or particular difficulties associated with abnormal intraoperative bleeding resulting from the increased bleeding tendency induced by tirofiban therapy, and immediate postoperative brain CTs did not show ventriculostomy-related hemorrhage.

Operations such as EVD placement or decompressive craniectomy are sometimes necessary after coiling, especially in cases of ruptured cerebral aneurysms. With $\mathrm{SAH}$, our experience suggests that tirofiban may not expose patients to a higher risk of hemorrhagic complications, especially those undergoing invasive procedures such as placement of an EVD.

In our study, infarction developed in 2 patients. One patient was admitted with a history of headache and mild left-sided weakness. The patient was treated with stent-assisted coiling for a posterior communicating artery aneurysm, and immediate postprocedural angiography showed no in-stent thrombosis and normal patency of the anterior choroidal artery. Left-sided weakness im- proved gradually after stent-assisted coiling. Initial brain CT did not show an abnormality in the internal capsule, but a follow-up brain MR imaging 7 days after the procedure revealed infarction in the posterior limb of the internal capsule. Considering the clinical course of left-sided weakness, vasospasm may contribute to infarction. Another possible mechanism is flow disturbance due to the stent. The stent was deployed across the origin of the anterior choroidal artery. Therefore, another possibility as a potential cause is disturbance of blood flow to the anterior choroidal artery by the stent struts. The other patient who was treated for an anterior communicating artery aneurysm developed an infarction in the left frontal lobe. However, follow-up angiography showed normal patency of the intracranial vessel. A possible mechanism is distal emboli into the anterior cerebral artery related to the stent. Additionally, the anterior communicating artery aneurysm had a broad neck, so the exposure of the coil mass alone could contribute to distal emboli into the anterior cerebral artery. Vasospasm of the anterior cerebral artery associated with SAH may be another possible mechanism but is less likely.

Despite infarction and hemorrhagic complications, 34 (85\%) patients had a good outcome (GOS of 4 or 5), with only 2 patients $(5 \%)$ having a GOS of $\leq 2$ at discharge in this study. In addition, our mortality rate of only $2.5 \%$ highlights the potential benefits of tirofiban in stent-assisted coiling of ruptured intracranial aneurysms.

As an alternative to clopidogrel in patients with acute coronary syndromes, the novel platelet P2Y12 inhibitors, prasugrel and ticagrelor, have been evaluated. Both agents provide stronger and more consistent platelet inhibition than clopidogrel. ${ }^{27,28}$ The time to onset of maximal platelet inhibition after administration of loading doses of prasugrel and ticagrelor is 30 minutes and 2 hours, respectively, and both agents are orally administered. ${ }^{28,29}$ We believe that the more rapid onset of the antiplatelet effect with tirofiban is more suited to acute intraprocedural decision-making, for example, when intraoperative coil prolapse necessitates the use of a stent. Moreover, intravenous administration of the drug may be more convenient and may decrease the risk of aspiration in patients with decreased consciousness.

In recent years, there has been an increase in the use of flow diverters for intracranial aneurysms. However, because there is no immediate aneurysm occlusion after stent implantation, the need for antiplatelet therapy complicates the use of flow diverters for acute ruptured aneurysms. The treatment of ruptured aneurysms with a flow diverter has been reported, but there are still concerns related to the risk of recurrent $\mathrm{SAH}$ during antiplatelet therapy while remodeling is underway. ${ }^{30,31}$ Further study regarding a viable acute antiplatelet agent for potential flow-diverter cases is important.

Our study had some limitations. The retrospective and singlearm design of our study may limit it because we did not compare aspirin and clopidogrel with glycoprotein IIb/IIIa receptor antagonists. Therefore, we are unable to determine whether the combination of aspirin and clopidogrel has any clinical advantage over glycoprotein IIb/IIIa receptor antagonists or vice-versa. In addition, the dosage and method of tirofiban administration during coiling of intracranial aneurysms were based on the standard application of the drug for acute coronary syndrome. When we first used tirofiban (in March 2010) for stent-assisted coiling in SAH, 
we could not find any articles about tirofiban as a premedication for stent-assisted coiling of acutely ruptured intracranial aneurysms. We, therefore, chose to use the cardiac dose because data on the use of tirofiban at this dose were available. Later, several studies suggested a protocol of $0.1-\mu \mathrm{g} / \mathrm{kg} / \mathrm{min}$ maintenance infusion without a loading dose for stent placement of intracranial aneurysms. $^{20,32}$ The pharmaceutical company Merck \& Co. recommended that the infusion should be continued through angiography and for 12-24 hours after angioplasty or atherectomy. ${ }^{33}$ Tirofiban infusion was, therefore, continued for 12-24 hours after the procedure in our study. However, a postprocedural bolus and initiation of aspirin and clopidogrel (Plavix) could be a viable alternative method. Further studies to research the optimal dose and application method of the drug are needed.

The small sample size limits some of the conclusions that can be drawn. More data are necessary to determine whether tirofiban increases the risk of hemorrhage after EVD placement.

\section{CONCLUSIONS}

Tirofiban is a fast-acting, fast-deactivated, highly selective nonpeptide glycoprotein IIb/IIIa antagonist. In our study, tirofiban showed a low risk of symptomatic hemorrhagic or thromboembolic complications. Thus, tirofiban may offer a safe and effective alternative as an antiplatelet premedication during stent-assisted coiling of acutely ruptured intracranial aneurysms. Larger and randomized trials are needed to further clarify this observation.

\section{REFERENCES}

1. Molyneux AJ, Kerr RS, Yu LM, et al. International subarachnoid aneurysm trial (ISAT) of neurosurgical clipping versus endovascular coiling in 2143 patients with ruptured intracranial aneurysm: a randomised comparison of effects on survival, dependency, seizures, rebleeding, subgroups, and aneurysm occlusion. Lancet 2005;366:809-17 CrossRef Medline

2. Benitez RP, Silva MT, Klem J, et al. Endovascular occlusion of widenecked aneurysms with a new intracranial microstent (Neuroform) and detachable coils. Neurosurgery 2004;54:1359-67; discussion 1368 CrossRef Medline

3. Fiorella D, Albuquerque FC, Han P, et al. Preliminary experience using the Neuroform stent for the treatment of cerebral aneurysms. Neurosurgery 2004;54:6-16; discussion 16-17 CrossRef Medline

4. Piotin M, Blanc R, Spelle L, et al. Stent-assisted coiling of intracranial aneurysms: clinical and angiographic results in 216 consecutive aneurysms. Stroke 2010;41:110-15 CrossRef Medline

5. Jeong HW, Jin SC. Intra-arterial infusion of a glycoprotein IIb/IIIa antagonist for the treatment of thromboembolism during coil embolization of intracranial aneurysm: a comparison of abciximab and tirofiban. AJNR Am J Neuroradiol 2013;34:1621-25 CrossRef Medline

6. Aggour M, Pierot L, Kadziolka K, et al. Abciximab treatment modalities for thromboembolic events related to aneurysm coiling. $\mathrm{Neu}$ rosurgery 2010;67(2 suppl operative):503-08 CrossRef Medline

7. Yi HJ, Gupta R, Jovin TG, et al. Initial experience with the use of intravenous eptifibatide bolus during endovascular treatment of intracranial aneurysms. AJNR Am J Neuroradiol 2006;27:1856-60 Medline

8. Dawson P, Strickland NH. Thromboembolic phenomena in clinical angiography; role of materials and technique. J Vasc Interv Radiol 1991;2:125-32 CrossRef Medline

9. Pierot L, Spelle L, Vitry F. Immediate clinical outcome of patients harboring unruptured intracranial aneurysms treated by endovascular approach: results of the ATENA study. Stroke 2008;39:2497504 CrossRef Medline
10. Bodily KD, Cloft HJ, Lanzino G, et al. Stent-assisted coiling in acutely ruptured intracranial aneurysms: a qualitative, systematic review of the literature. AJNR Am J Neuroradiol 2011;32:1232-36 CrossRef Medline

11. Meckel S, Singh TP, Undrén P, et al. Endovascular treatment using predominantly stent-assisted coil embolization and antiplatelet and anticoagulation management of ruptured blood blister-like aneurysms. AJNR Am J Neuroradiol 2011;32:764-71 CrossRef Medline

12. Ries $\mathrm{T}$, Buhk JH, Kucinski $\mathrm{T}$, et al. Intravenous administration of acetylsalicylic acid during endovascular treatment of cerebral aneurysms reduces the rate of thromboembolic events. Stroke 2006;37: 1816-21 CrossRef Medline

13. Müller I, Seyfarth M, Rüdiger S, et al. Effect of a high loading dose of clopidogrel on platelet function in patients undergoing coronary stent placement. Heart 2001;85:92-93 CrossRef Medline

14. Park JH, Kim JE, Sheen SH, et al. Intraarterial abciximab for treatment of thromboembolism during coil embolization of intracranial aneurysms: outcome and fatal hemorrhagic complications. J Neurosurg 2008;108:450-57 CrossRef Medline

15. Walsh RD, Barrett KM, Aguilar MI, et al. Intracranial hemorrhage following neuroendovascular procedures with abciximab is associated with high mortality: a multicenter series. Neurocrit Care 2011; 15:85-95 CrossRef Medline

16. Cho YD, Lee JY, Seo JH, et al. Early recurrent hemorrhage after coil embolization in ruptured intracranial aneurysms. Neuroradiology 2012;54:719-26 CrossRef Medline

17. Tcheng JE, Ellis SG, George BS, et al. Pharmacodynamics of chimeric glycoprotein IIb/IIIa integrin antiplatelet antibody Fab 7E3 in high-risk coronary angioplasty. Circulation 1994;90: 1757-64 CrossRef Medline

18. Dart RC, ed, McCuigan MA, Caravati EM. Medical Toxicology. 3rd ed. Philadelphia: Lippincott, Williams \& Wilkins; 2003:635-37

19. Highlights of prescribing information: AGGRASTAT (tirofiban hydrochloride). Food and Drug Administration, Silver Spring, Maryland. http://www.accessdata.fda.gov/drugsatfda_docs/label/ 2013/020912s019s020lbl.pdf. Accessed August 7, 2015

20. Chalouhi N, Jabbour P, Kung D, et al. Safety and efficacy of tirofiban in stent-assisted coil embolization of intracranial aneurysms. $\mathrm{Neu}$ rosurgery 2012;71:710-14; discussion 714 CrossRef Medline

21. Lodi YM, Latorre JG, El-Zammar Z, et al. Stent assisted coiling of the ruptured wide necked intracranial aneurysm. J Neurointerv Surg 2012;4:281-86 CrossRef Medline

22. Roitberg BZ, Khan N, Alp MS, et al. Bedside external ventricular drain placement for the treatment of acute hydrocephalus. $\mathrm{Br} J$ Neurosurg 2001;15:324-27 CrossRef Medline

23. Ehtisham A, Taylor S, Bayless L, et al. Placement of external ventricular drains and intracranial pressure monitors by neurointensivists. Neurocrit Care 2009;10:241-47 CrossRef Medline

24. Binz DD, Toussaint LG 3rd, Friedman JA. Hemorrhagic complications of ventriculostomy placement: a meta-analysis. Neurocrit Care 2009;10:253-56 CrossRef Medline

25. Scholz C, Hubbe U, Deininger M, et al. Hemorrhage rates of external ventricular drain (EVD), intracranial pressure gauge (ICP) or combined EVD and ICP gauge placement within $48 \mathrm{~h}$ of endovascular coil embolization of cerebral aneurysms. Clin Neurol Neurosurg 2013;115:1399-402 CrossRef Medline

26. Kung DK, PoliceniBA, Capuano AW, et al. Risk of ventriculostomyrelated hemorrhage in patients with acutely ruptured aneurysms treated using stent-assisted coiling. J Neurosurg 2011;114:1021-27 CrossRef Medline

27. Wiviott SD, Braunwald E, McCabe CH, et al; TRITON-TIMI 38 Investigators. Prasugrel versus clopidogrel in patients with acute coronary syndromes. $N$ Engl J Med 2007;357:2001-15 CrossRef Medline

28. Gurbel PA, Bliden KP, Butler K, et al. Randomized double-blind assessment of the ONSET and OFFSET of the antiplatelet effects of ticagrelor versus clopidogrel in patients with stable coronary artery 
disease: the ONSET/OFFSET study. Circulation 2009;120:2577-85 CrossRef Medline

29. Zhu B, Effron MB, Kulkarni MP, et al. The onset of inhibition of platelet aggregation with prasugrel compared with clopidogrel loading doses using gatekeeping analysis of integrated clinical pharmacology data. J Cardiovasc Pharmacol 2011;57:317-24 CrossRef Medline

30. Kulcsár Z, Wetzel SG, Augsburger L, et al. Effect of flow diversion treatment on very small ruptured aneurysms. Neurosurgery 2010;67: 789-93 CrossRef Medline
31. Aydin K, Arat A, Sencer S, et al. Treatment of ruptured blood blisterlike aneurysms with flow diverter SILK stents. J Neurointerv Surg 2015;7:202-09 CrossRef Medline

32. Chalouhi N, Zanaty M, Starke R, et al. O-022: a new protocol for anticoagulation with tirofiban during stenting of intracranial aneurysms. J Neurointerv Surg 2014;6:A11-12 CrossRef

33. AGGRASTAT (tirofiban hydrochloride). MERCK \& CO., INC., West Point, PA. Food and Drug Administration, Silver Spring, Maryland. http://www.accessdata.fda.gov/drugsatfda_docs/label/1998/20913lbl. pdf. Accessed August 8, 2015 